\title{
Intracycle interference in the interaction of laser and electron beams
}

\author{
Yuya Morimoto $\odot^{*}$ and Peter Hommelhoff $\odot$ \\ Laser Physics, Department of Physics, Friedrich-Alexander-Universität Erlangen-Nürnberg (FAU), \\ Staudtstraße 1, 91058, Erlangen, Germany
}

(Received 21 May 2020; accepted 28 September 2020; published 16 October 2020)

\begin{abstract}
A high-energy electron beam coupled to an optical field experiences a quantized energy modulation by multiples of the photon energy, originating from the temporal periodicity of the optical field by virtue of intercycle interference. Here we investigate the quantum interference occurring at shorter timescales, namely, within half an optical cycle, which is called intracycle interference. We show that the sub-half-cycle energy and phase modulation are imprinted into and largely modulate electron energy spectra regardless of the laser pulse duration. The intracycle effect is universal, meaning that it shows up for almost any electron velocity and optical wavelength. It thus facilitates coherent control of free-electron beams in microscopes or accelerators with a resolution of below an optical cycle, i.e., on attosecond timescales. The results obtained also apply to multiphoton and attosecond physics in atoms, molecules, and solids.
\end{abstract}

DOI: 10.1103/PhysRevResearch.2.043089

\section{INTRODUCTION}

Efficient coupling between a nonrelativistic electron beam and an optical field occurs in the presence of a third body which satisfies energy-momentum conservation. An early example is laser-assisted electron scattering, in which electrons scattered by atomic or molecular targets in a laser field absorb or emit photons [1-4]. For the manipulation of energetic electron beams, a semi-infinite laser field created by a nanometer-thick membrane provided the spatially homogeneous coupling for electrons passing through it [5-8]. Optical near fields induced by laser irradiation at a nanotip, grating, prism surface, or nanofabricated silicon structure have offered a suitable platform for energy modulation of nanofocused electron beams in electron microscopes [9-12]. These all-optical schemes now form the basis of photon-induced near-field electron microscopy [9], diffractive imaging of ultrafast reactions [4,7], generation and characterization of attosecond electron pulses [7,13-17], dielectric-laser accelerators [12,16-18], free-electron quantum optics [10,15], and quantum control of radiation and excitation processes $[19,20]$.

The appearance of discrete photon peaks in the electron energy spectra is explained by the extended wave-packet nature of the electrons in a beam. When the temporal coherence of the electrons is longer than an optical cycle $T=2 \pi / \omega$, with $\omega$ the angular frequency, the cycle-periodic acceleration or deceleration of electrons causes the interference in the energy spectrum with a spacing of $\hbar \omega$. While this intercycle interference and the appearance of photon peaks have recently been extensively studied $[9,10,21,22]$, quantum interference

\footnotetext{
*yuya.morimoto@fau.de

Published by the American Physical Society under the terms of the Creative Commons Attribution 4.0 International license. Further distribution of this work must maintain attribution to the author(s) and the published article's title, journal citation, and DOI.
}

associated with different timescales has not been sufficiently investigated.

In this work, we examine the quantum interference occurring within a half cycle of the laser field, so within a period $<T / 2$. The concept of the intracycle effect is shown in Fig. 1. An electron pulse [blue in Fig. 1(a)] at a subrelativistic velocity interacts with a laser field (red) in the presence of a third body. In classical mechanics, the electron experiences a time-dependent energy modulation following the oscillation of the laser field [Figs. 1(a) and 1(e)]. When we focus on half an optical cycle at around $t=0$ [red dotted line in Fig. 1(e)], there are pairs of points in time giving the same amount of the acceleration, marked by black circles and green arrows. The electrons separated in time by less than $T / 2$ and accelerated by the same amount can interfere with each other when we measure an energy spectrum. We note that this discussion has close relevance to strongly driven electrons at atoms, molecules, and solids, and especially to the laser-assisted Auger decay [23-25].

\section{THEORETICAL MODEL}

We perform quantum mechanical simulations to explore the intracycle effect quantitatively. An electron pulse [blue in Fig. 1(a)], resembling the ones in in ultrafast electron microscopes $[8,10,16]$, is described as a statistical ensemble of coherent wave packets. We assume that each wave packet has the same coherence time $\tau_{e}$, in full width at half maximum (FWHM), which approximately gives the coherent energy bandwidth of $\sim 4 \ln 2 \hbar / \tau_{e}$, where $\hbar$ is the reduced Planck constant. The statistical ensemble has an incoherent energy spread $\Delta_{E}$ and envelope duration $\Delta_{t}$. We fix these parameters as $\Delta_{E}=0.5 \mathrm{eV}$ and $\Delta_{t}=500 \mathrm{fs}$, both in FWHM. We consider normal distributions for all these parameters. Because the electron's longitudinal momentum is usually much larger than its transversal momentum, we describe the wave packet as a superposition of plane waves propagating along the $z$ direction [see Fig. 1(a)] and consider one-dimensional dynamics. When 
(a)
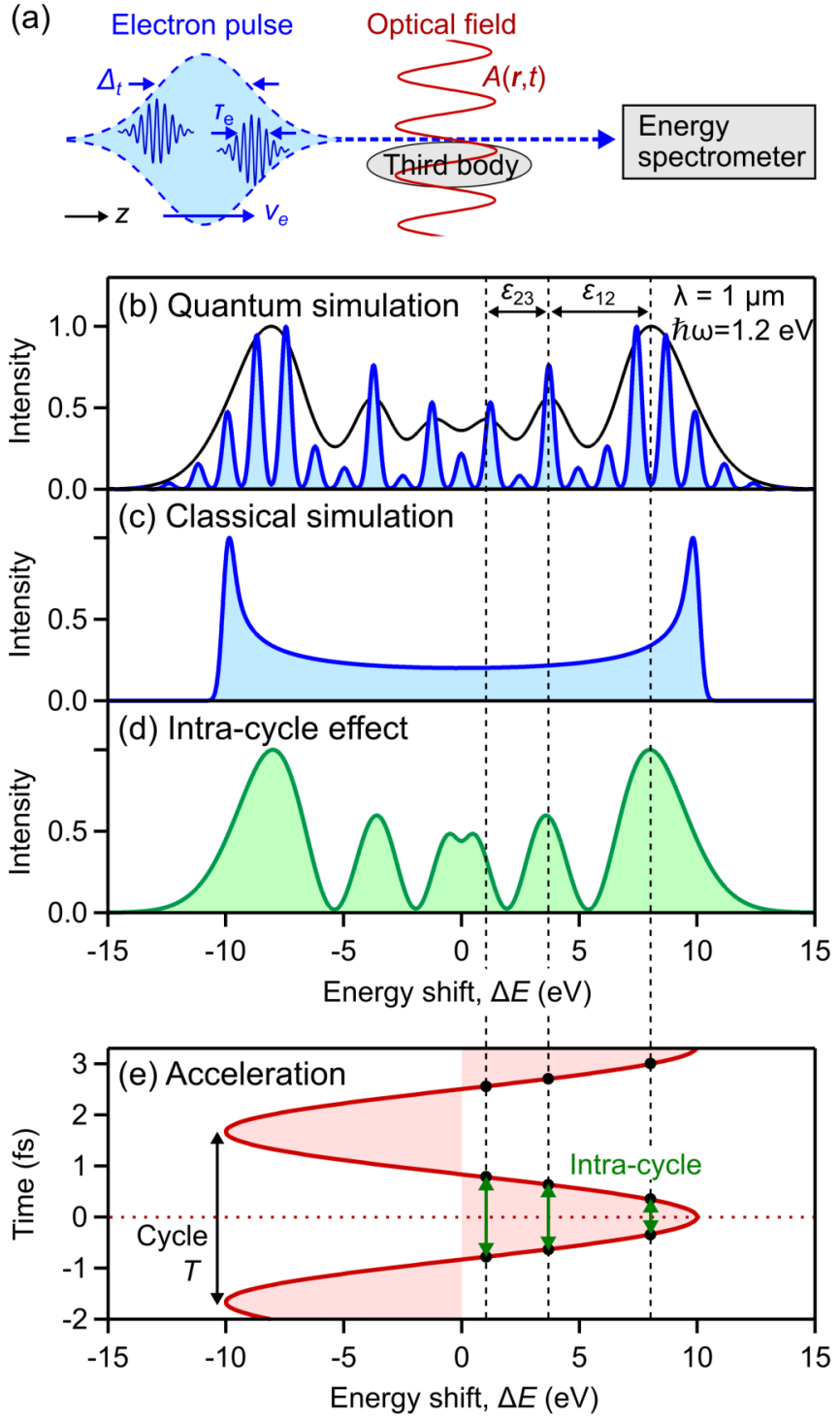

FIG. 1. Concept of intracycle interference. (a) Schematic of laser-electron beam interaction. An electron pulse (blue) propagating along the $z$ axis is modulated by an optical field (red) in the presence of a third body. An energy spectrum after the interaction is calculated. (b) Results of quantum mechanical simulations with Eq. (2) at $\lambda=1 \mu \mathrm{m}$ and $\Delta E_{\max }=10 \mathrm{eV}$. Blue curve: $0.5-\mathrm{eV}$ resolution, black curve: $1.8-\mathrm{eV}$ resolution. (c) Result of the classical simulation. (d) Intracycle interference as given by Eq. (3). (e) Time-dependent acceleration and intracycle interference. See text for details.

we write an electron wave packet interacting with the optical field as $\Psi(z, t)=\phi(z, t) \exp \left(i p_{e} z / \hbar-i E_{e} t / \hbar\right)$, where $p_{e}$ and $E_{e}$ are the electron's central longitudinal momentum and energy, respectively, the envelope function $\phi(z, t)$ is given by $[8,21]$

$$
\begin{aligned}
& \phi(z, t) \\
& \quad=\phi_{0}\left(z-v_{e} t\right) \exp \left[i \frac{q_{e} p_{e}}{\hbar m_{e}} \int_{-\infty}^{t} A_{z}\left(z+v_{e} t^{\prime}-v_{e} t, t^{\prime}\right) d t^{\prime}\right],
\end{aligned}
$$

where $q_{e}$ and $m_{e}$ are the electron charge and rest mass, respectively, $v_{e}$ is the central velocity, $A_{z}(z, t)$ is the $z$ component of the vector potential of the optical field, and $\phi_{0}\left(z-v_{e} t\right)=\left(2 \pi \sigma_{z}^{2}\right)^{-1 / 4} \exp \left[-\left(z-v_{e} t\right)^{2} / 4 \sigma_{z}^{2}\right]$ is the initial envelope function with $\sigma_{z}=v_{e} \tau_{e} / 2 \sqrt{2 \ln (2)}$. To derive Eq. (1), we assumed the minimum coupling Hamiltonian, Coulomb gauge $(\boldsymbol{\nabla} \cdot \boldsymbol{A}=0)$, a small momentum shift compared to the incident momentum, and a negligibly small wave-packet dispersion during the interaction [21]. We note that the interaction between the incident laser field and the third body is included in the vector potential $A_{z}[8,21]$. The field amplitude here is of the order of $1 \mathrm{~V} / \mathrm{nm}[9,10,16]$, corresponding to the intensity of $10^{11} \mathrm{~W} / \mathrm{cm}^{2}$. The associated ponderomotive energy is merely of the order of $0.01 \mathrm{eV}$ at 1- $\mu \mathrm{m}$ wavelength and therefore neglected in Eq. (1) for high-energy electrons. In classical mechanics, the time derivative of the phase inside the exponential function corresponds to the kinetic energy shift. Therefore, Eq. (1) indicates that the amount of energy shift follows the oscillation of the vector potential $A_{z}$. For the monochromatic optical field $A_{z}(z, t)=A_{z, \text { env }}(z, t) \cos [\varphi(z)-\omega t]$, where $A_{z, \text { env }}(z, t)$ is the slowly varying space-time envelope and $\varphi(z)$ is the spatial phase, we can simplify Eq. (1) after the interaction $(z, t \rightarrow$ $+\infty)$ as

$\phi(z, t)=\phi_{0}\left(z-v_{e} t\right) \exp \left(i \frac{\Delta E_{\max }}{\hbar \omega} \sin \left[\omega\left(\frac{z}{v_{\mathrm{e}}}-t\right)+\tilde{\varphi}\right]\right)$,

where $\Delta E_{\max }$ is the classically allowed maximum energy gain and $\tilde{\varphi}$ is a constant phase; see Appendix A for details. The maximal energy gain $\Delta E_{\max }$ is proportional to the field amplitude. An energy spectrum of a wave packet is obtained from $\left|\Psi_{p}\right|^{2}$, where $\Psi_{p}$ is the momentum-space wave function given by the Fourier transform of $\Psi(z, t)$ after the interaction $(t \rightarrow$ $+\infty)$. We compute $\left|\Psi_{p}\right|^{2}$ for the wave packets forming the electron pulse and take their incoherent sum. For an electron pulse longer than an optical cycle $\left(\Delta_{t}>T\right)$, the energy spectra are independent of the phase $\tilde{\varphi}$, and therefore we set $\tilde{\varphi}=0$ in this work. Throughout the rest of this article, we assume a 30-keV incident electron beam. However, we note that Eq. (2) yields energy spectra nearly independent of the velocity $v_{e}$ at a fixed $\Delta E_{\max }$, as long as the light-driven energy shift is much smaller than the incident kinetic energy. The sinusoidal phase modulation in Eq. (2) allows us to express it with a series of Bessel functions of the first kind, as shown in previous works $[8,10,19,21,22]$. The close connection between the sinusoidal phase modulation and the Bessel function can also be found in the pioneering theoretical works of more than a half century ago $[26,27]$ and in recent experiments even without free electrons [28,29], showing the generality of the inter- and intracycle effects. It is worth mentioning that the intracycle interference can also be seen in the simulated spectra of the above-threshold ionization with an assumption of the half collision using the Volkov wave function [30] and in the spectra of laser-assisted Auger decay [23-25]. However, the high-energy electron beam we consider here provides the ideal platform because its energy spectrum purely captures the interaction between free electrons and an optical field, free from the other processes such as ionization. We keep the form 
of Eq. (2) in order to give the analytical form of the intracycle effect below.

\section{RESULTS AND DISCUSSION}

\section{A. Numerical results}

We first consider a monochromatic optical field and electron wave packets of high temporal coherence $\left(\tau_{e}=\Delta_{t}=\right.$ $500 \mathrm{fs}$ ) using Eq. (2). The blue curve in Fig. 1(b) shows simulation results for $\lambda=1 \mu \mathrm{m}(\hbar \omega=1.24 \mathrm{eV})$ and $\Delta E_{\max }=$ $10 \mathrm{eV}$. In addition to photon peaks separated by $\hbar \omega$, we observe a modulation with longer periods, peaked at energy shifts of $\Delta E= \pm 1.1, \pm 3.7$, and $\pm 8.1 \mathrm{eV}$. The interference becomes clearer with the poorer energy resolution $\left(\Delta_{E}=\right.$ $1.5 \hbar \omega)$, which does not suffice to resolve photon peaks, as shown by the black curve. To confirm that the observed longperiod interference is a quantum mechanical effect, we show in Fig. 1(c) the result of a classical simulation [31]. The classical result shows a smooth profile and maxima only at $\Delta E= \pm 9.8 \mathrm{eV}$. The classical energy spectrum is given by the histogram of the optical field amplitude [Fig. 1(e)] [31]; hence it has two maxima close to the cutoff energies, where the field changes slowly in time. This comparison confirms that the long-period energy modulation seen in Fig. 1(b) is indeed due to a quantum mechanical effect. The energy spacings between these peaks are more than two times larger than the photon energy, suggesting that the interference is associated with a timescale less than half an optical cycle, so it is intracycle. When we compare the energy spacings between the peaks $\varepsilon_{12}$ and $\varepsilon_{23}$ defined in Fig. 1(b), we see that the spacings are not identical $\left(\varepsilon_{12}=4.4 \mathrm{eV}\right.$ and $\varepsilon_{23}=2.6 \mathrm{eV}$, respectively) and the spacing at smaller gain $\left(\varepsilon_{23}\right)$ is shorter than that at higher gain $\left(\varepsilon_{12}\right)$. This indicates that the interference appearing at smaller energy gain is related with longer timescale, in line with the green arrows in Fig. 1(e).

The wavelength dependence gives more insight into the intracycle effect. The left panels in Fig. 2(a) show numerically obtained energy spectra from Eq. (2) at wavelengths of $\lambda=2$, 5 , and $10 \mu \mathrm{m}$ at a fixed maximum energy gain of $\Delta E_{\max }=$ $10 \mathrm{eV}$; compare also with Fig. 1(b). Because the energy spectra with continuous-wave optical fields are symmetric around zero energy gain, we show only the results of the positive energy shift. At $\lambda=5$ and $10 \mu \mathrm{m}$, the photon peaks are not resolved because of their small photon energies $(\hbar \omega=0.25$ and $0.12 \mathrm{eV}$, respectively) compared to the incoherent energy spread $\left(\Delta_{E}=0.5 \mathrm{eV}\right)$. With increasing wavelength, the fringe spacing becomes shorter, reflecting the fact that the field cycle becomes longer. The longest fringe spacing $\left[\varepsilon_{12}\right.$; see Fig. 1(b) for definition] equals $4.4,2.5,1.5$, and $1.0 \mathrm{eV}$ at $\lambda=1,2,5$, and $10 \mu \mathrm{m}$, respectively, suggesting a $\sim \lambda^{-3 / 2}$ dependence (see below for more discussions).

\section{B. Analytical formula}

We now investigate the intracycle effect for electrons with low temporal coherence, in contrast to the simulations above assuming long coherence times. Figure 2(b) compares simulations at $\lambda=5 \mu \mathrm{m}$ ( $T=16.7 \mathrm{fs})$ with four different temporal coherence times $\tau_{e}=T, \frac{T}{2}, \frac{T}{4}$, and $\frac{T}{8}$, from the top. When $\tau_{e} \geqslant T / 2$, there are no significant differences in the energy
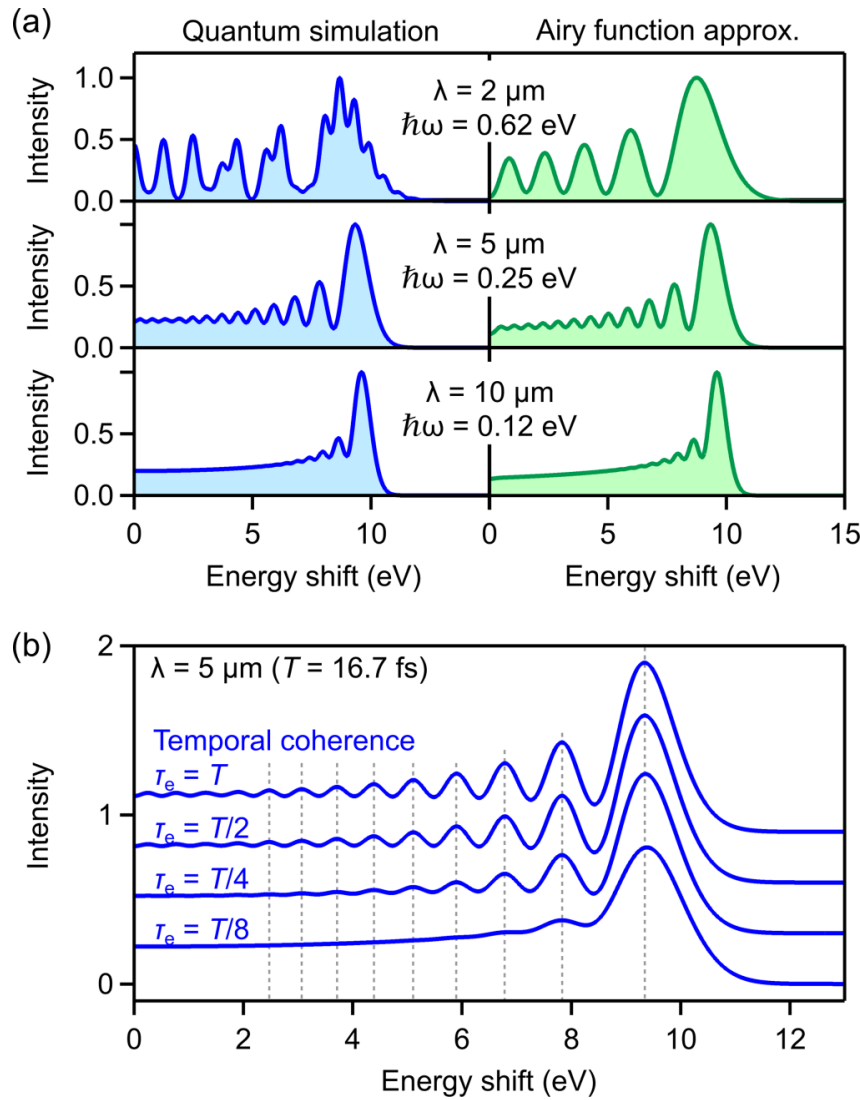

FIG. 2. (a) Wavelength dependence. Left panels: results of quantum mechanical simulations with Eq. (2) at $\lambda=2,5$, and $10 \mu \mathrm{m}$. Right panels: intracycle interference given by Eq. (3). (b) Influence of the electron wave packet's temporal coherence. Results of the quantum mechanical simulation are plotted for $\tau_{\mathrm{e}}=T, \frac{T}{2}, \frac{T}{4}$, and $\frac{T}{8}$ from the top. The baselines are shifted vertically for clarity.

spectra, suggesting that the interference occurs within half an optical cycle. When the temporal coherence is shorter than $\frac{T}{2}$, the interference contrast becomes lower and vanishes at smaller energy shifts. At $\tau_{e}=\frac{T}{8}$, we can see interference only at the energy shift close to the cutoff energy. All these results further support the intracycle interference picture.

We now derive an analytical form of the intracycle effect. Because the intracycle interference is associated with the two different times symmetric around the timing for maximum energy gain or loss [see Fig. 1(e)], we expand the sine function in Eq. (2) by its Taylor series at around $z / v_{e}-t=0$ (red dotted line) and obtain $\sin \left[\omega\left(\frac{z}{v_{e}}-t\right)\right] \approx$ $\omega\left(\frac{z}{v_{e}}-t\right)-\frac{\omega^{3}}{6}\left(\frac{z}{v_{e}}-t\right)^{3}$. We include here only the first two terms, up to the third order. By this expansion, the vector potential and the phase modulation are not periodic anymore and the photon peaks do not show up in the energy spectra. We note in passing that the cubic phase term of a matter wave has also recently been found and investigated in entirely different systems, such as classical surface-gravity water waves [32], in atom interferometry [33] and in nondiffracting electron beams [34]. It is known to be related with the Airy function of the first kind $\operatorname{Ai}(x)[32,35,36]$. Indeed, we obtain the energy spectrum 
as

$$
\left|\Psi_{p}\right|^{2}=C\left|\operatorname{Ai}\left(\left[\frac{2}{(\hbar \omega)^{2} \Delta E_{\max }}\right]^{\frac{1}{3}}\left(|\Delta E|-\Delta E_{\max }\right)\right)\right|^{2},
$$

where $C$ is a normalization constant; see Appendix A for details. Notably, the spectral shape of the intracycle interference does not depend on the incident electron velocity $v_{e}$ at a given maximum energy gain $\Delta E_{\max }$. The dependence on the optical frequency $\omega^{2 / 3}$ comes from the Taylor expansion above, related to how the amount of energy shift changes with time at around the maximum gain or loss. The interference patterns given by Eq. (3) are plotted with green curves and compared with quantum simulations in Figs. 1(d) and 2(a), after having been convolved with the statistical energy spread of $\Delta_{E}=0.5 \mathrm{eV}$. The overall shape of the spectra and the peak positions of the quantum simulations are well reproduced by Eq. (3); see black dotted lines in Figs. 1(b) and 1(d). Because we take only the lowest two orders of the expansion, the agreement is better at higher energy gain/loss parts.

We can quantify the intracycle fringe spacings. Because the magnitude of the Airy function $|\operatorname{Ai}(x)|$ has the first and second maxima at $x_{1}=-1.019$ and $x_{2}=-3.249$ (hence $x_{1}-x_{2}=$ 2.230) the largest energy spacing $\left[\varepsilon_{12}\right.$; see Fig. 1(b) is given by

$$
\varepsilon_{12}=2.230\left[\frac{(\hbar \omega)^{2} \Delta E_{\max }}{2}\right]^{\frac{1}{3}} .
$$

This equation indicates that the fringe spacing follows $\omega^{2 / 3}$ or $\lambda^{-2 / 3}$, as heuristically obtained above. Figure 3(a) shows the wavelength dependence from $\lambda=0.5$ to $15 \mu \mathrm{m}$ at the fixed maximum energy gain $\left(\Delta E_{\max }\right)$ of $100 \mathrm{eV}$. The results of the quantum simulation with Eq. (2) (blue circles) are reproduced almost perfectly by Eq. (4) shown by the green curve. For comparison, we plot a $\lambda^{-1}$ curve by the black broken line. The agreement is poor with the $\lambda^{-1}$ dependence, further supporting the validity of Eq. (4). The dependence on the maximum energy gain $\left(\Delta E_{\max }\right)$ is weak, following $\Delta E_{\max }^{1 / 3}$. We show the maximum gain dependence in Fig. 3(b) for $\lambda=2$ and $5 \mu \mathrm{m}$. All the quantum mechanical results plotted by blue squares are well reproduced by Eq. (4) shown by the green curves. Equation (4) tells us that the intracycle fringe spacing is normally larger than the photon energy. Even at the small limit of $\Delta E_{\max }=\hbar \omega$, we obtain $\varepsilon_{12}=1.8 \hbar \omega$.

\section{Space-time averaging and energy modulation by single-cycle fields}

We have demonstrated that intracycle interference occurs at almost any optical wavelength and field strength. The intracycle fringes can be observed even with an electron beam whose temporal coherence and energy spread are not sufficient to resolve photon peaks. However, there have been no clear experimental reports so far. We attribute this to the spatial and temporal averaging effect. In contrast to the photon peaks whose locations are independent of the field amplitude, the intracycle interference is affected by the spatiotemporal field strength according to Eq. (3). Therefore, spatially uniform optical fields with comparably long pulse durations are ideal to clearly observe the intracycle interference
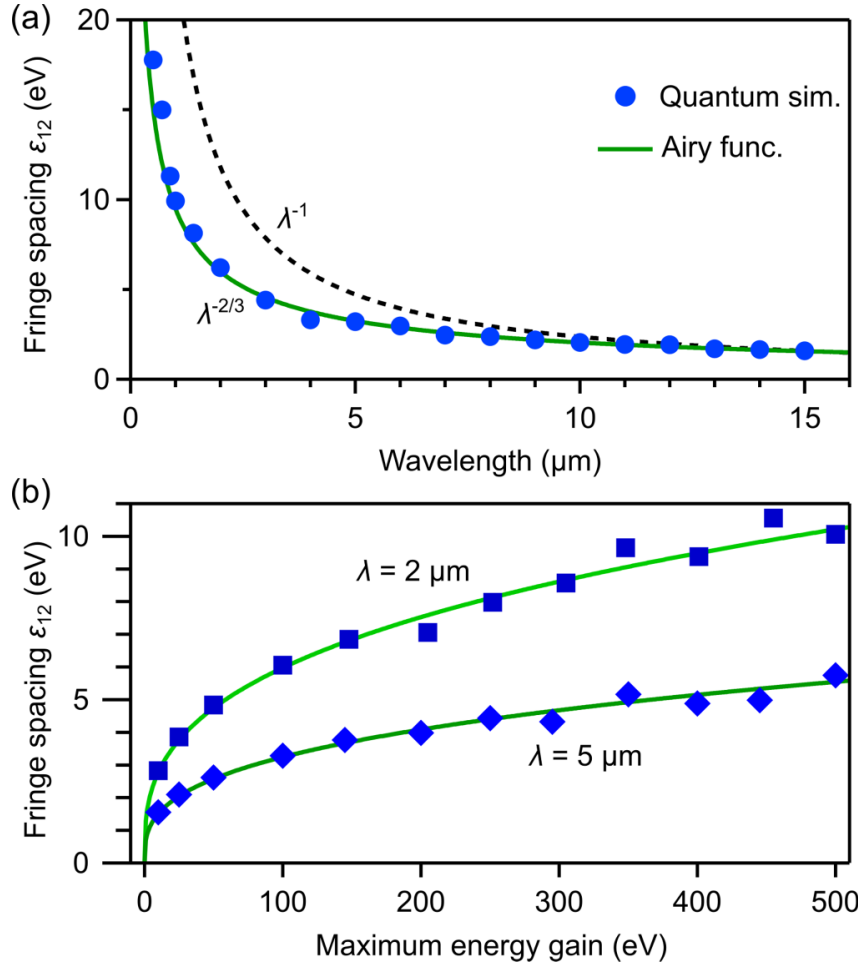

FIG. 3. Intracycle fringe spacing. (a) Wavelength dependence. Results of quantum mechanical simulations [Eq. (2)] at $\Delta E_{\max }=$ $100 \mathrm{eV}$ (blue circles) are compared with the analytical result (green curve) of Eq. (4). The black dashed line shows a $\lambda^{-1}$ dependence for comparison, normalized at $\lambda=15 \mu \mathrm{m}$. (b) Maximum energy gain $\left(\Delta E_{\max }\right)$ dependence. Simulation results for $\lambda=2$ and $5 \mu \mathrm{m}$ (blue squares) are compared with the analytical results (green curves).

effects discussed. Indeed, recent results using nanofocused ultrashort electron beams by Ropers et al. [10,15] contain signals which might be assigned to the intracycle effect but were interpreted in a different way, based on Rabi oscillation.

An interesting exception to avoid the temporal averaging is the realm of ultrashort pulses of few- or single-cycle duration [37]. Such extremely short optical pulses have peak cycles with significantly different amplitudes from the others. Therefore, the electrons accelerated or decelerated by a peak cycle are isolated in the energy spectrum. Figure 4 shows energy spectra induced by single-cycle fields of carrier wavelength $\lambda=1 \mu \mathrm{m}$ at $\Delta E_{\max }=100 \mathrm{eV}$ and $\tau_{e}=10 \mathrm{fs}$, given by Eq. (1) for a spatially and spectrally uniform interaction; see Appendix B for details. For the sinelike optical field shown in Fig. 4(a), we observe a symmetric spectrum. At large energy shifts of $|\Delta E|>65 \mathrm{eV}$, highlighted by green boxes, we observe clear intracycle interferences. There are no photon peaks $(\hbar \omega=1.24 \mathrm{eV})$ appearing because no other cycles exist to give the same acceleration or deceleration. The inner part of the spectrum $(|\Delta E|<65 \mathrm{eV})$ shows the combination of the intracycle and intercycle interferences. In contrast, the cosinelike field in Fig. 4(b) yields an asymmetric energy spectrum. The intracycle interference driven by the peak cycle can be seen above $40 \mathrm{eV}$, as highlighted by the green box. Clearly, the waveform has dramatic consequences for the spectra (see 


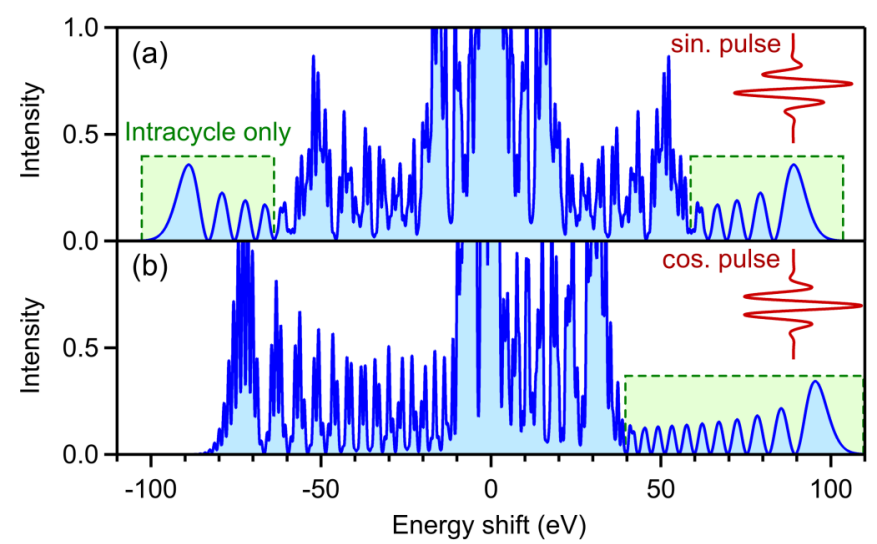

FIG. 4. Single-cycle-driven electron energy modulation. (a) Energy spectrum of electrons modulated by a sinelike waveform with a carrier wavelength of $\lambda=1 \mu \mathrm{m}$. (b) Result with a cosinelike field. Near the cutoff energies induced by the peak cycles (green boxes), clear intracycle interference is observed without intercycle photon peaks.

also Fig. 5 in the Appendix). These results demonstrate the capability of controlling intra- and intercycle interferences by optical field waveforms designed on the single-cycle level.

\section{CONCLUSION}

In this work, we have introduced and investigated analytically and numerically the concept of intracycle interference in the interaction between an optical field and a high-energy electron beam. We showed that the intracycle effect is approximately represented by a cubic phase term, which leads to an Airy function behavior in the energy spectrum. The cubic phase term is imprinted on an extremely short timescale and is fully tunable with the optical wavelength and the interaction strength. Combined with the tailored optical fields provided by today's ultrashort pulse lasers, we foresee that the coherent control of electron beams with sub-half-cycle precision may provide opportunities in various branches of electron beam applications. Examples include free-electron quantum optics $[10,15]$, ultrafast electron microscopes [7,9], phase-contrast imaging [38], free-electron lasers [39], coherent control of radiation and excitation processes $[19,20]$, and dielectric-laser acceleration [12,16-18]. Similarly, we expect renewed interest in quantum interference in the physics of above-threshold ionization [30].

\section{ACKNOWLEDGMENTS}

This work was supported by the Gordon and Betty Moore Foundation (GBMF) through Grant No. GBMF4744 "Accelerator on a Chip International Program-ACHIP" and FAU Emerging Talents Initiative. We thank Peter Baum and Philip Dienstbier for helpful discussions.

\section{APPENDIX A: THEORETICAL DETAILS}

We derive Eqs. (2) and (3) in the main text. With $A_{z}(z, t)=$ $A_{z, \text { env }}(z, t) \cos [\varphi(z)-\omega t]$, the exponential part of Eq. (1) be-

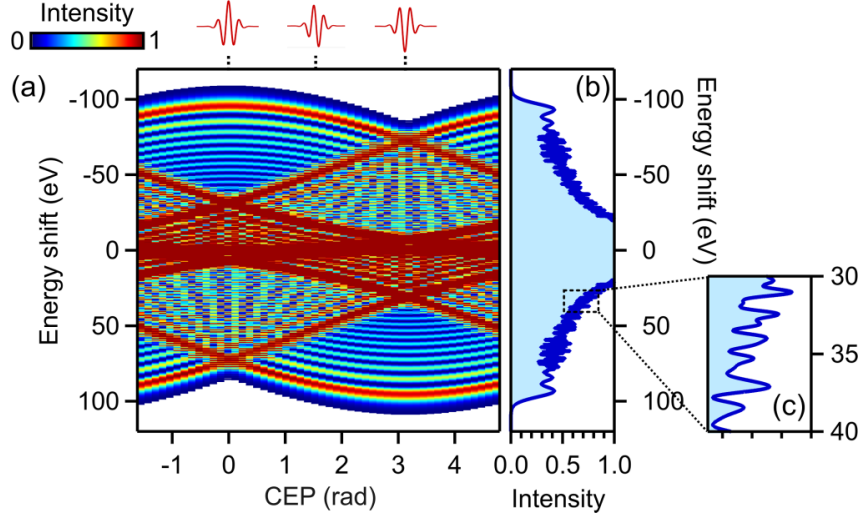

FIG. 5. (a) Simulated electron energy spectra as a function of carrier-envelope phase $\left(\mathrm{CEP}, \varphi_{\mathrm{CEP}}\right)$. Zero CEP corresponds to the cosinelike waveform. The arclike interference fringes originate from the intracycle effect. (b) CEP-averaged energy spectrum. (c) Magnified view of the dashed black box in (b). Photon peaks are resolved only in the magnified plot.

comes

$$
\begin{aligned}
K= & \exp \left[i \frac{q_{e} p_{e}}{\hbar m_{e}} \int_{-\infty}^{t} A_{z}\left(z+v_{e} t^{\prime}-v_{e} t, t^{\prime}\right) d t^{\prime}\right] \\
= & \exp \left(i \frac{q_{e} p_{e}}{\hbar m_{e}} \int_{-\infty}^{t} A_{z, \text { env }}\left(z+v_{e} t^{\prime}-v_{e} t, t^{\prime}\right)\right. \\
& \left.\times \cos \left[\varphi\left(z+v_{e} t^{\prime}-v_{e} t\right)-\omega t^{\prime}\right] d t^{\prime}\right) \\
= & \exp \left(i \frac{q_{e} p_{e}}{\hbar m_{e}} \int_{-\infty}^{z} A_{z, \operatorname{env}}\left(z^{\prime}, \frac{z^{\prime}-z}{v_{e}}+t\right)\right. \\
& \left.\times \cos \left[\varphi\left(z^{\prime}\right)-\omega \frac{z^{\prime}-z}{v_{e}}-\omega t\right] \frac{d z^{\prime}}{v_{e}}\right) \\
= & \exp \left(i \frac { q _ { e } p _ { e } } { \hbar m _ { e } v _ { e } } \operatorname { R e } \left[\exp \left(\mathrm{i} \omega \frac{z}{v_{e}}-\mathrm{i} \omega t\right)\right.\right. \\
& \left.\left.\times \int_{-\infty}^{z} A_{z, \operatorname{env}}\left(z^{\prime}, \frac{z^{\prime}-z}{v_{e}}+t\right) e^{i \varphi\left(z^{\prime}\right)-i \omega \frac{z^{\prime}}{v_{e}}} d z^{\prime}\right]\right) .
\end{aligned}
$$

When we express the integral by its magnitude and argument,

$\int_{-\infty}^{z} A_{z, \text { env }}\left(z^{\prime}, \frac{z^{\prime}-z}{v_{e}}+t\right) e^{i \varphi\left(z^{\prime}\right)} e^{-i \omega \frac{z^{\prime}}{v_{e}}} d z^{\prime}=|B(z)| e^{i \tilde{\varphi}(z)-\frac{i \pi}{2}}$,

the exponential term becomes

$$
K=\exp \left(i \frac{q_{e} p_{e}}{\hbar m_{e} v_{e}}|B(z)| \sin \left[\omega\left(\frac{z}{v_{e}}-t\right)+\tilde{\varphi}(z)\right]\right) .
$$

Because the optical field becomes zero at $z \rightarrow+\infty(t \rightarrow$ $+\infty),|B(z)|$ and $\tilde{\varphi}(z)$ converge to constant values at the limit. Since the partial derivative of the term inside of the exponential function in time represents the energy shift, we can write $\frac{q_{e} p_{e}}{\hbar m_{e} v_{e}}|B(z)|_{z \rightarrow+\infty}=\Delta E_{\max } / \hbar \omega$. We then obtain

$$
K=\exp \left(i \frac{\Delta E_{\max }}{\hbar \omega} \sin \left[\omega\left(\frac{z}{v_{e}}-t\right)+\tilde{\varphi}\right]\right),
$$

where $\tilde{\varphi}$ is the argument $\tilde{\varphi}(z)$ at the limit of $z \rightarrow+\infty$. 
By substituting the Taylor expansion of the sine function into Eq. (2), we obtain

$$
\Psi(z, t)=\phi_{0}\left(z-v_{e} t\right) \exp \left[i \frac{\Delta E_{\max }}{\hbar}\left(\frac{z}{v_{e}}-t\right)-i \frac{\Delta E_{\max } \omega^{2}}{6 \hbar}\left(\frac{z}{v_{e}}-t\right)^{3}\right] \exp \left(\frac{i}{\hbar} p_{e} z-\frac{i}{\hbar} E_{e} t\right) .
$$

The momentum-space wave function $\Psi_{p}$ is given by [19]

$$
\Psi_{p}=\frac{1}{\sqrt{2 \pi \hbar}} \int_{-\infty}^{+\infty} \Psi(z, t) \exp \left(-\frac{i}{\hbar} p z+\frac{i}{\hbar} E_{p} t\right) d z
$$

at the limit of $t \rightarrow+\infty$. Because we are interested in the ultrafast dynamics within half an optical cycle, we can assume that the envelope function is constant; i.e., $\phi_{0}\left(z-v_{e} t\right)=1$. With this assumption, the momentum-space wave function is given by

$$
\begin{aligned}
\Psi_{p} & =\frac{1}{\sqrt{2 \pi \hbar}} \int_{-\infty}^{+\infty} \exp \left[i \frac{\Delta E_{\max }}{\hbar}\left(\frac{z}{v_{e}}-t\right)-i \frac{\Delta E_{\max } \omega^{2}}{6 \hbar}\left(\frac{z}{v_{e}}-t\right)^{3}\right] \exp \left(\frac{i}{\hbar} p_{e} z-\frac{i}{\hbar} E_{e} t\right) \exp \left(-\frac{i}{\hbar} p z+\frac{i}{\hbar} E_{p} t\right) d z \\
& =\frac{1}{\sqrt{2 \pi \hbar}} \exp \left[\frac{i}{\hbar}\left(E_{p}-E_{e}\right) t\right] \int_{-\infty}^{+\infty} \exp \left[i \frac{\Delta E_{\max }}{\hbar}\left(\frac{z}{v_{e}}-t\right)-i \frac{\Delta E_{\max } \omega^{2}}{6 \hbar}\left(\frac{z}{v_{e}}-t\right)^{3}\right] \exp \left[\frac{i}{\hbar}\left(p_{e}-p\right) z\right] d z \\
& =\frac{1}{\sqrt{2 \pi \hbar}} \exp \left(\frac{i}{\hbar}\left[E_{p}-E_{e}-v_{e}\left(p-p_{e}\right)\right] t\right) \int_{-\infty}^{+\infty} \exp \left(i \frac{1}{\hbar v_{e}}\left[\Delta E_{\max }-v_{e}\left(p-p_{e}\right)\right] z^{\prime}-i \frac{\Delta E_{\max } \omega^{2}}{6 \hbar v_{e}^{3}} \mathrm{z}^{\prime 3}\right) d z^{\prime} \\
& =-\frac{1}{\sqrt{2 \pi \hbar}}\left(\frac{2 \hbar v_{e}^{3}}{\Delta E_{\max } \omega^{2}}\right)^{1 / 3} \exp \left[\frac{i}{\hbar}\left(E_{p}-E_{e}-\Delta E\right) t\right] \int_{-\infty}^{+\infty} \exp \left[i\left(\Delta E-\Delta E_{\max }\right)\left(\frac{2}{\Delta E_{\max } \hbar^{2} \omega^{2}}\right)^{1 / 3} z^{\prime \prime}+i \frac{\mathrm{z}^{\prime \prime 3}}{3}\right] d z^{\prime \prime},
\end{aligned}
$$

where we replaced $v_{e}\left(p-p_{e}\right)$ by the kinetic energy shift $\Delta E$ because we are considering a high-energy electron beam whose momentum shift due to the photon absorption or emission is much smaller than the initial momentum, $\left|p-p_{e}\right| \ll$ $p_{e}$. By using the definition of the Airy function of the first kind,

$$
\operatorname{Ai}(x)=\frac{1}{2 \pi} \int_{-\infty}^{+\infty} \exp \left(i x z+i \frac{z^{3}}{3}\right) d z
$$

we obtain

$$
\left|\Psi_{p}\right|^{2}=C\left|\operatorname{Ai}\left[\left(\frac{2}{(\hbar \omega)^{2} \Delta E_{\max }}\right)^{\frac{1}{3}}\left(\Delta E-\Delta E_{\max }\right)\right]\right|^{2},
$$

with a constant $C$. On the other hand when we consider the Taylor expansion of the sine function at around a minimum energy shift peak, we get

$$
\left|\Psi_{p}\right|^{2}=C\left|\operatorname{Ai}\left[\left(\frac{2}{(\hbar \omega)^{2} \Delta E_{\max }}\right)^{\frac{1}{3}}\left(-\Delta E-\Delta E_{\max }\right)\right]\right|^{2} .
$$

\section{APPENDIX B: SINGLE-CYCLE-DRIVEN ENERGY MODULATION}

We assume the spatially and spectrally homogeneous interaction as in the scheme with a flat metallic membrane [5-8,30], where electrons exit from or enter an optical field when passing through it on attosecond timescales. It is known that in such a scheme, the amount of the energy modulation is determined by the vector potential at the timing when electrons pass through the membrane [5,6]. When the vector potential is given by a Fourier-transform-limited waveform of a Gaussian envelope, Eq. (A1) is expressed as

$$
\begin{aligned}
K= & \exp \left(i \frac{\Delta E_{\max }}{\hbar \omega} \exp \left[-2 \ln (2) \frac{\left(\frac{z}{v_{e}}-t\right)^{2}}{\tau^{2}}\right]\right. \\
& \left.\times \sin \left[\omega\left(\frac{z}{v_{e}}-t\right)+\varphi_{\mathrm{CEP}}\right]\right),
\end{aligned}
$$

where $\tau$ is the optical field duration in FWHM and $\varphi_{\mathrm{CEP}}$ is the carrier-envelope phase. Figure 5 shows the results of quantum mechanical simulations for single-cycle optical fields $(\tau=T)$ of the central wavelength of $\lambda=1 \mu \mathrm{m}$ as a function of $\varphi_{\mathrm{CEP}}$. We note that the phase of the vector potential is shifted by $\pi / 2$ from the phase term in (B1) and $\varphi_{\mathrm{CEP}}=0$ corresponds to the cosinelike field.
[1] D. Andrick and L. Langhans, J. Phys. B: At. Mol. Phys. 9, L459 (1976).

[2] A. Weingartshofer, J. K. Holmes, G. Caudle, E. M. Clarke, and H. Krüger, Phys. Rev. Lett. 39, 269 (1977).

[3] R. Kanya, Y. Morimoto, and K. Yamanouchi, Phys. Rev. Lett. 105, 123202 (2010).
[4] Y. Morimoto, R. Kanya, and K. Yamanouchi, Phys. Rev. Lett. 115, 123201 (2015).

[5] T. Plettner, R. L. Byer, E. Colby, B. Cowan, C. M. S. Sears, J. E. Spencer, and R. H. Siemann, Phys. Rev. Lett. 95, 134801 (2005). 
[6] F. O. Kirchner, A. Gliserin, F. Krausz, and P. Baum, Nat. Photonics 8, 52 (2013).

[7] Y. Morimoto and P. Baum, Nat. Phys. 14, 252 (2018).

[8] G. M. Vanacore, I. Madan, G. Berruto, K. Wang, E. Pomarico, R. J. Lamb, D. McGrouther, I. Kaminer, B. Barwick, F. J. García de Abajo, and F. Carbone, Nat. Commun. 9, 2694 (2018).

[9] B. Barwick, D. J. Flannigan, and A. H. Zewail, Nature 462, 902 (2009).

[10] A. Feist, K. E. Echternkamp, J. Schauss, S. V Yalunin, S. Schäfer, and C. Ropers, Nature 521, 200 (2015).

[11] S. Nehemia, R. Dahan, M. Shentcis, O. Reinhardt, Y. Adiv, K. Wang, O. Beer, Y. Kurman, X. Shi, M. H. Lynch, and I. Kaminer, arXiv:1909.00757.

[12] J. Breuer and P. Hommelhoff, Phys. Rev. Lett. 111, 134803 (2013).

[13] C. M. S. Sears, E. Colby, R. Ischebeck, C. McGuinness, J. Nelson, R. Noble, R. H. Siemann, J. Spencer, D. Walz, T. Plettner, and R. L. Byer, Phys. Rev. Spec. Top.-Accel. Beams 11, 061301 (2008).

[14] M. Kozák, N. Schönenberger, and P. Hommelhoff, Phys. Rev. Lett. 120, 103203 (2018)

[15] K. E. Priebe, C. Rathje, S. V. Yalunin, T. Hohage, A. Feist, S. Schäfer, and C. Ropers, Nat. Photonics 11, 793 (2017).

[16] N. Schönenberger, A. Mittelbach, P. Yousefi, J. McNeur, U. Niedermayer, and P. Hommelhoff, Phys. Rev. Lett. 123, 264803 (2019).

[17] D. S. Black, U. Niedermayer, Y. Miao, Z. Zhao, O. Solgaard, R. L. Byer, and K. J. Leedle, Phys. Rev. Lett. 123, 264802 (2019).

[18] E. A. Peralta, K. Soong, R. J. England, E. R. Colby, Z. Wu, B. Montazeri, C. McGuinness, J. McNeur, K. J. Leedle, D. Walz, E. B. Sozer, B. Cowan, B. Schwartz, G. Travish, and R. L. Byer, Nature 503, 91 (2013).

[19] Y. Pan and A. Gover, Phys. Rev. A 99, 052107 (2019).

[20] A. Gover and A. Yariv, Phys. Rev. Lett. 124, 064801 (2020).

[21] S. T. Park, M. Lin, and A. H. Zewail, New J. Phys. 12, 123028 (2010).

[22] F. J. García de Abajo, A. Asenjo-Garcia, and M. Kociak, Nano Lett. 10, 1859 (2010).

[23] A. K. Kazansky, I. P. Sazhina, and N. M. Kabachnik, Phys. Rev. A 82, 033420 (2010).
[24] M. Meyer, P. Radcliffe, T. Tschentscher, J. T. Costello, A. L. Cavalieri, I. Grguras, A. R. Maier, R. Kienberger, J. Bozek, C. Bostedt, S. Schorb, R. Coffee, M. Messerschmidt, C. Roedig, E. Sistrunk, L. F. Di Mauro, G. Doumy, K. Ueda, S. Wada, S. Düsterer et al., Phys. Rev. Lett. 108, 063007 (2012).

[25] A. A. Gramajo, R. Della Picca, S. D. López, and D. G. Arbó, J. Phys. B: At., Mol. Opt. Phys. 51, 055603 (2018).

[26] L. S. Brown and T. W. B. Kibble, Phys. Rev. 133, A705 (1964).

[27] F. V. Bunkin and M. V. Fedorov, Sov. Phys. JETP 22, 844 (1966).

[28] K. C. Miao, A. Bourassa, C. P. Anderson, S. J. Whiteley, A. L. Crook, S. L. Bayliss, G. Wolfowicz, G. Thiering, P. Udvarhelyi, V. Ivády, H. Abe, T. Ohshima, Á. Gali, and D. D. Awschalom, Sci. Adv. 5, eaay0527 (2019).

[29] P. Imany, N. B. Lingaraju, M. S. Alshaykh, D. E. Leaird, and A. M. Weiner, Sci. Adv. 6, eaba8066 (2020).

[30] J. Javanainen, J. H. Eberly, and Q. Su, Phys. Rev. A 38, 3430 (1988).

[31] Y. Morimoto and P. Baum, Phys. Rev. A 97, 033815 (2018).

[32] G. G. Rozenman, M. Zimmermann, M. A. Efremov, W. P. Schleich, L. Shemer, and A. Arie, Phys. Rev. Lett. 122, 124302 (2019).

[33] O. Amit, Y. Margalit, O. Dobkowski, Z. Zhou, Y. Japha, M. Zimmermann, M. A. Efremov, F. A. Narducci, E. M. Rasel, W. P. Schleich, and R. Folman, Phys. Rev. Lett. 123, 083601 (2019).

[34] N. Voloch-Bloch, Y. Lereah, Y. Lilach, A. Gover, and A. Arie, Nature 494, 331 (2013).

[35] G. A. Siviloglou, J. Broky, A. Dogariu, and D. N. Christodoulides, Phys. Rev. Lett. 99, 213901 (2007).

[36] A. K. Kazansky, I. P. Sazhina, V. L. Nosik, and N. M. Kabachnik, J. Phys. B: At., Mol. Opt. Phys. 50, 105601 (2017).

[37] Y. Morimoto and P. Baum, arXiv:2004.11776 [Phys. Rev. Lett. (to be published)].

[38] O. Schwartz, J. J. Axelrod, S. L. Campbell, C. Turnbaugh, R. M. Glaeser, and H. Müller, Nat. Methods 16, 1016 (2019).

[39] P. K. Maroju, C. Grazioli, M. Di Fraia, M. Moioli, D. Ertel, H. Ahmadi, O. Plekan, P. Finetti, E. Allaria, L. Giannessi, G. De Ninno, C. Spezzani, G. Penco, S. Spampinati, A. Demidovich, M. B. Danailov, R. Borghes, G. Kourousias, C. E. Sanches Dos Reis, F. Billé et al., Nature 578, 386 (2020). 